https://doi.org/10.46344/JBINO.2020.v09i03.12

\title{
EFFICACY OF INSECTICIDES AND BOTANICALS AGAINST BREVICORYNE BRASSICAE POPULATION ON CABBAGE
}

\section{HENRY DEBBARMA ${ }^{1}$ AND VIJAY KUMAR*2}

'Department of Entomology, V.C.S.G Uttarakhand University of Horticulture \& Forestry, Bharsar- 246123, Pauri Garhwal (Uttarakhand), India

2Department of Plant Pathology, V.C.S.G Uttarakhand University of Horticulture \& Forestry, Bharsar- 246123, Pauri Garhwal (Uttarakhand), India

\section{ABSTRACT}

The investigation was conducted on the "Efficacy of insecticides and botanicals against Brevicoryne brassicae population on cabbage" at field of Vegetable Research and Demonstration Block, College of Horticulture, VCSG UUHF, Bharsar during 2018. Among the insecticides Imidacloprid $17.8 \%$ SL $(0.0356 \%)$, Thiamethoxam 25\% WG $(0.0025 \%)$, Spinosad 45\% SC (0.0144\%) and Acetamiprid 20\% SP $(0.002 \%)$ were found effective against $B$. brassicae whereas, Novaluron 10\% EC (0.0075\%) was found least effective against the pest (i.e. 57.15 and 19.12 per cent change over control at the end of $1^{\text {st }}$ and $2^{\text {nd }}$ spray, respectively). In botanicals Neem leaf extract + Cow urine (5\%) was observed highly effective (i.e. 32.29 and 8.59 per cent change over control at the end of $1^{\text {st }}$ and $2^{\text {nd }}$ spray, respectively) and Lantana leaf extract + Cow urine (5\%) was observed least effective against B. brassicae (i.e. 22.08 and 6.02 per cent change over control at 15 days after both spray, respectively).

Keywords: Cabbage, Brevicoryne brassicae, Efficacy, Insecticides, Botanicals 


\section{INTRODUCTION}

Cabbage (Brassica oleracea var capitata) is the most popular vegetable grown throughout the world which belongs to the family Cruciferae and genus Brassica. Cabbages are a highly nutritious food source and contain a high amount of minerals and vitamins like $A$, B1, B2 and C (Hasan and Solaiman., 2012). Cabbage covers about $4.3 \%$ of total area under vegetables in India (Vanitha et al., 2013) and in cabbage production India is next to China.

In India, a total of 37 insect pests have been reported to feed on cabbage (Lal., 1975). The extent of damage due to these pests in India is known to range from 7 to 90 per cent with consequent reduction in yield from 20 to 80 per cent on cabbage (Prasad., 1963). Among these pests, Cabbage aphids (Brevicoryne brassicae) are one of the most important pests of cabbage. Aphids feed by sucking sap from their host plants and continued feeding by aphids causes yellowing wilting and stunting of plants (Opfer and Mcgrath., 2013). Aphids infested plants show slow growth, which results in 35-75\% yield losses (Khan et al., 2015).

\section{Methodology}

The experiment was conducted in the Vegetable Research and Demonstration Block, Department of Vegetable Science, College of Horticulture, VCSG Uttarakhand University of Horticulture and Forestry, Bharsar, Pauri Garhwal with ten treatments and three replications in RCBD (Randomized Complete Block Design).
To reduce insect pest infestation, various insecticides are applied and those insecticides could lead to problem of the insect resistance, environmental and food contamination and reduced populations of natural enemies which may result in secondary pest outbreaks or pest resurgences (Garratt and Kennedy., 2006). Therefore use of alternatives including botanicals, bio pesticides and new insecticides is essential to reduce pest resurgences. Among the new insecticides Thiamethoxam, Imidacloprid and Acetamiprid are the important neonicotinoids insecticides (Maienfisch et al., 1999). Iwasa et al. (2004) reported that Imidacloprid is a fastest acting neonicotinoid insecticide for controlling sucking insects. On the other hand several workers were reported on the use of plant extract and cow urine for the control of insect pests of field crops (Dubey et al., 2004 and Sharma et al., 2009). In botanical plant extracts Azadirachta indica, Lantana camera and Eupatorium spp. are found promising to manage cabbage aphid (Sood et al., 2000). The efficacy of cow urine in combination with neem extract is found highly effective in aphid (Gupta., 2005).

The botanicals and insecticides of required concentration were prepared in water just before spraying during evening. All botanical leave extracts were prepared by soaking $100 \mathrm{~g}$ chopped leaves in 1 litter cow urine for 15 days before application and the insecticidal solution was prepared by using following formula: 


$$
\mathbf{v}=\frac{\mathrm{C} \times \mathrm{A}}{\% a \cdot i .}
$$

Where,

$\mathrm{V}=$ Volume of the insecticide

$C=$ Concentration required

$A=$ Amount of spray solution needed

\%a.i. = Per cent of active ingredient of the insecticide

The spraying was done by using hand sprayer fitted with hollow cone nozzle. The first spray was given on 03thjune 2018 and the second spray was on 19thjune 2018. Numbers of insects in cabbage field were recorded at 1 day before spray and $3,7,11$ and 15 days after both spray. Percent reduction of insect over control was recorded according to the following formula

$$
\text { PROC\% }=\left(1-\frac{T a \times C b}{T b \times C a}\right) \times 100
$$

Where,

PROC = Population reduction over control

Ta $=$ Population of insects after treatment application

$\mathrm{Tb}=$ Population of insects before treatment application

$\mathrm{Ca}=$ Population of insects in control after treatment application

$\mathrm{Cb}=$ Population of insects in control before treatment application

\section{Treatments Details:}

Nine treatments along with control were evaluated against cabbage aphids (B. brassicae) on cabbage. The details of treatment were given in Table 1.

Table No 1: Details of different insecticides and botanicals evaluated against cabbage aphids.

\begin{tabular}{|c|l|c|}
\hline T.No. & \multicolumn{1}{|c|}{ Treatments } & Dose (\%) \\
\hline $\mathrm{T}_{1}$ & Control & - \\
\hline $\mathrm{T}_{2}$ & Neem leaf extract + Cow urine & 5 \\
\hline $\mathrm{T}_{3}$ & Lantana leaf extract + Cow urine & 5 \\
\hline $\mathrm{T}_{4}$ & Parthaniun leaf extract + Cow urine & 5 \\
\hline $\mathrm{T}_{5}$ & Eupatorium leaf extract + Cow urine & 0.0075 \\
\hline $\mathrm{T}_{6}$ & Novaluron 10\% EC & 0.0025 \\
\hline $\mathrm{T}_{7}$ & Thiamethoxam 25\% WG & 0.0356 \\
\hline $\mathrm{T}_{8}$ & Imidacloprid 17.8\% SL & 0.0144 \\
\hline $\mathrm{T}_{9}$ & Spinosad 45\% SC & 0.002 \\
\hline $\mathrm{T}_{10}$ & Acetamiprid 20\% SP & 5 \\
\hline
\end{tabular}

Impact of various treatments on cabbage aphid after first spray

The data obtained after first spray are presented in Table No 2 and Table No 3. After first spray all the treatments were found effective against cabbage aphid compared with untreated control. Among the insecticides the minimum aphid population was recorded in plot treated with Imidacloprid $17.8 \%$ SL with 97.54, 
82.26, 74.32 and 90.62 aphids per plant at 3, 7, 11 and 15 days after spray, respectively, followed by the plot treated with Thiamethoxam 25\% WG and Acetamiprid $20 \%$ SP. The per cent changes in aphid population are also observed highest (i.e. 64.35, 70.78, 74.89 and 70.52 per cent reduction over control at 3, 7, 11 and 15 days after spray) in the plot treated with Imidacloprid $17.8 \%$ SL. Spinosad $45 \%$ SC was also found effective against aphid with 114.33, 98.32, 88.95 and 102.67 aphids per plant (i.e. 49.14, 57.48, 63.42 and 59.34 per cent reduction over control) at 3, 7, 11 and 15 days after spray, respectively. Neem leaf extract + Cow urine was found highly effective among the botanicals with 112.67, 125.92, 133.00 and 142.33 aphids per plant at $3,7,11$ and 15 days after spray, respectively, and the per cent change was also found highest on it. Parthaniun leaf extract + Cow urine and Eupatorium leaf extract + Cow urine were observed quit effective against aphid compared with Lantana leaf extract + Cow urine.

Table No. 2: Efficacy of different insecticides and botanicals against cabbage aphid after first spray

\begin{tabular}{|c|c|c|c|c|c|c|c|}
\hline \multirow{3}{*}{ T. No. } & \multirow{3}{*}{ Treatments } & \multirow{3}{*}{$\begin{array}{c}\text { Dose } \\
(\%)\end{array}$} & \multicolumn{5}{|c|}{ No. of cabbage aphid/ plant } \\
\hline & & & \multirow{2}{*}{$\begin{array}{c}\text { Pre } \\
\text { spray } \\
\text { count }\end{array}$} & \multicolumn{4}{|c|}{ After $1^{\text {st }}$ application } \\
\hline & & & & 3 DAS & 7 DAS & 11 DAS & 15 DAS \\
\hline $\mathrm{T}_{1}$ & Control & & $\begin{array}{l}225.67 \\
(15.06)\end{array}$ & $\begin{array}{l}231.67 \\
(15.25)\end{array}$ & $\begin{array}{l}238.31 \\
(15.47)\end{array}$ & $\begin{array}{l}250.56 \\
(15.86) \\
\end{array}$ & $\begin{array}{l}260.19 \\
(16.16) \\
\end{array}$ \\
\hline $\mathrm{T}_{2}$ & Neem leaf extract + Cow urine & 5 & $\begin{array}{l}182.33 \\
(13.54)\end{array}$ & $\begin{array}{l}112.67 * \\
(10.66)\end{array}$ & $\begin{array}{l}125.92 * \\
(11.26)\end{array}$ & $\begin{array}{l}133.00^{*} \\
(11.57)\end{array}$ & $\begin{array}{l}142.33 * \\
(11.97)\end{array}$ \\
\hline $\mathrm{T}_{3}$ & Lantana leaf extract + Cow urine & 5 & $\begin{array}{l}203.67 \\
(14.29)\end{array}$ & $\begin{array}{l}\text { 150.00* } \\
(12.28)\end{array}$ & $\begin{array}{l}166.32 * \\
(12.93)\end{array}$ & $\begin{array}{l}173.29 * \\
(13.20)\end{array}$ & $\begin{array}{l}182.66^{*} \\
(13.55)\end{array}$ \\
\hline $\mathrm{T}_{4}$ & Parthaniun leaf extract + Cow urine & 5 & $\begin{array}{l}215.00 \\
(14.69)\end{array}$ & $\begin{array}{l}147.67 * \\
(12.19)\end{array}$ & $\begin{array}{l}165.08^{*} \\
(12.88)\end{array}$ & $\begin{array}{l}171.67 * \\
(13.14)\end{array}$ & $\begin{array}{l}181.25^{*} \\
(13.50)\end{array}$ \\
\hline $\mathrm{T}_{5}$ & Eupatorium leaf extract + Cow urine & 5 & $\begin{array}{l}189.33 \\
(13.79)\end{array}$ & $\begin{array}{l}137.00^{*} \\
(11.74)\end{array}$ & $\begin{array}{l}146.79 * \\
(12.15)\end{array}$ & $\begin{array}{l}157.20^{*} \\
(12.57)\end{array}$ & $\begin{array}{l}165.99 * \\
(12.92)\end{array}$ \\
\hline $\mathrm{T}_{6}$ & Novaluron $10 \%$ EC & 0.0075 & $\begin{array}{l}210.00 \\
(14.52)\end{array}$ & $\begin{array}{l}116.67^{*} \\
(10.84)\end{array}$ & $\begin{array}{l}100.43^{*} \\
(10.07)\end{array}$ & $\begin{array}{l}90.28^{*} \\
(9.55)\end{array}$ & $\begin{array}{l}103.75^{*} \\
(10.23)\end{array}$ \\
\hline $\mathrm{T}_{7}$ & Thiamethoxam 25\% WG & 0.0025 & $\begin{array}{l}251.33 \\
(15.89)\end{array}$ & $\begin{array}{l}97.74^{*} \\
(9.93)\end{array}$ & $\begin{array}{l}80.74 * \\
(9.04)\end{array}$ & $\begin{array}{l}78.19^{*} \\
(8.89)\end{array}$ & $\begin{array}{l}92.50 * \\
(9.67)\end{array}$ \\
\hline $\mathrm{T}_{8}$ & Imidacloprid $17.8 \%$ SL & 0.0356 & $\begin{array}{l}266.67 \\
(16.36)\end{array}$ & $\begin{array}{l}97.54^{*} \\
(9.92)\end{array}$ & $\begin{array}{l}82.26^{*} \\
(9.12)\end{array}$ & $\begin{array}{l}74.32 * \\
(8.67)\end{array}$ & $\begin{array}{l}90.62^{*} \\
(9.57)\end{array}$ \\
\hline $\mathrm{T}_{9}$ & Spinosad $45 \% \mathrm{SC}$ & 0.0144 & $\begin{array}{l}219.00 \\
(14.83) \\
\end{array}$ & $\begin{array}{l}114.33^{*} \\
(10.73)\end{array}$ & $\begin{array}{l}98.32^{*} \\
(9.96) \\
\end{array}$ & $\begin{array}{l}88.95^{*} \\
(9.48)\end{array}$ & $\begin{array}{l}102.67 * \\
(10.18)\end{array}$ \\
\hline $\mathrm{T}_{10}$ & Acetamiprid 20\% SP & 0.002 & $\begin{array}{l}238.67 \\
(15.48) \\
\end{array}$ & $\begin{array}{l}109.60^{*} \\
(10.51)\end{array}$ & $\begin{array}{r}97.93^{*} \\
(9.95) \\
\end{array}$ & $\begin{array}{r}86.17 * \\
(9.33) \\
\end{array}$ & $\begin{array}{l}99.42^{*} \\
(10.02) \\
\end{array}$ \\
\hline & $\mathrm{SE}(\mathrm{d})$ & - & 0.915 & 1.22 & 1.08 & 0.85 & 1.01 \\
\hline & C.D. $(0.05)$ & - & 1.93 & 2.58 & 2.28 & 1.80 & 2.12 \\
\hline
\end{tabular}

DAS- days after spray, ( ) = Values in parentheses are square root transformed value, *Significant at $5 \%$ level of significance compared with control 
Table No. 3: Change in cabbage aphid population after first spray.

\begin{tabular}{|c|l|c|c|c|c|c|}
\hline \multirow{2}{*}{ T.No. } & \multicolumn{2}{|c|}{ Treatments } & \multirow{2}{*}{$\begin{array}{c}\text { Dose } \\
(\%)\end{array}$} & \multicolumn{3}{|c|}{ Per cent change in aphid population } \\
\cline { 4 - 7 } & & & \multicolumn{3}{|c|}{ after first spray } \\
\hline $\mathrm{T}_{1}$ & Control & - & $\begin{array}{c}0.00 \\
(0.00)\end{array}$ & $\begin{array}{c}0.00 \\
(0.00)\end{array}$ & $\begin{array}{c}0.00 \\
(0.00)\end{array}$ & $\begin{array}{c}0.00 \\
(0.00)\end{array}$ \\
\hline $\mathrm{T}_{2}$ & Neem leaf extract + Cow urine & 5 & $\begin{array}{c}39.81^{*} \\
(39.10)\end{array}$ & $\begin{array}{c}34.60^{*} \\
(36.01)\end{array}$ & $\begin{array}{c}34.48^{*} \\
(35.94)\end{array}$ & $\begin{array}{c}32.29^{*} \\
(34.61)\end{array}$ \\
\hline $\mathrm{T}_{3}$ & Lantana leaf extract + Cow urine & 5 & $\begin{array}{c}28.14^{*} \\
(32.01)\end{array}$ & $\begin{array}{c}22.53^{*} \\
(28.32)\end{array}$ & $\begin{array}{c}23.24^{*} \\
(28.81)\end{array}$ & $\begin{array}{c}22.08^{*} \\
(28.00)\end{array}$ \\
\hline $\mathrm{T}_{4}$ & Parthaniun leaf extract + Cow urine & 5 & $\begin{array}{c}33.13^{*} \\
(35.12)\end{array}$ & $\begin{array}{c}27.28^{*} \\
(31.47)\end{array}$ & $\begin{array}{c}28.07^{*} \\
(31.98)\end{array}$ & $\begin{array}{c}26.98^{*} \\
(31.28)\end{array}$ \\
\hline $\mathrm{T}_{5}$ & Eupatorium leaf extract + Cow urine & 5 & $\begin{array}{c}29.50^{*} \\
(32.87)\end{array}$ & $\begin{array}{c}26.56^{*} \\
(31.00)\end{array}$ & $\begin{array}{c}25.21^{*} \\
(30.12)\end{array}$ & $\begin{array}{c}23.95^{*} \\
(29.28)\end{array}$ \\
\hline $\mathrm{T}_{6}$ & Novaluron 10\% EC & 0.0075 & $\begin{array}{c}45.89^{*} \\
(42.62)\end{array}$ & $\begin{array}{c}54.72^{*} \\
(47.68)\end{array}$ & $\begin{array}{c}61.28^{*} \\
(51.49)\end{array}$ & $\begin{array}{c}57.15^{*} \\
(49.09)\end{array}$ \\
\hline $\mathrm{T}_{7}$ & Thiamethoxam 25\% WG & 0.0025 & $\begin{array}{c}62.12^{*} \\
(51.99)\end{array}$ & $\begin{array}{c}69.57^{*} \\
(56.50)\end{array}$ & $\begin{array}{c}71.97^{*} \\
(58.01)\end{array}$ & $\begin{array}{c}68.08^{*} \\
(55.57)\end{array}$ \\
\hline $\mathrm{T}_{8}$ & Imidacloprid 17.8\% SL & 0.0356 & $\begin{array}{c}64.35^{*} \\
(5332)\end{array}$ & $\begin{array}{c}70.78^{*} \\
(57.25)\end{array}$ & $\begin{array}{c}74.89^{*} \\
(59.90)\end{array}$ & $\begin{array}{c}70.52^{*} \\
(57.09)\end{array}$ \\
\hline $\mathrm{T}_{9}$ & Spinosad 45\% SC & 0.0144 & $\begin{array}{c}49.14^{*} \\
(44.49)\end{array}$ & $\begin{array}{c}57.48^{*} \\
(49.28)\end{array}$ & $\begin{array}{c}63.42^{*} \\
(52.76)\end{array}$ & $\begin{array}{c}59.34^{*} \\
(50.36)\end{array}$ \\
\hline $\mathrm{T}_{10}$ & Acetamiprid 20\% SP & 0.002 & $\begin{array}{c}55.26^{*} \\
(48.00)\end{array}$ & $\begin{array}{c}61.14^{*} \\
(51.41)\end{array}$ & $\begin{array}{c}67.48^{*} \\
(55.20)\end{array}$ & $\begin{array}{c}63.87^{*} \\
(53.03)\end{array}$ \\
\hline & SE(d) & - & 0.68 & 0.56 & 0.61 & 0.45 \\
\hline & C.D.(0.05) & - & 1.43 & 1.19 & 1.28 & 0.95 \\
\hline
\end{tabular}

DAS- days after spray, ()$=$ Values in parentheses are angular transformed value

\section{Impact of various treatments on cabbage aphid after second spray}

The data of second spray are presented in Table No 4 and Table No 5. After second spray among insecticides the highest efficacy was observed in the plot treated with Imidacloprid $17.8 \%$ SL i.e. 52.33, 32.92, 38.89 and 49.99 aphids per plant and the per cent reduction over control was 43.93, 66.23, 60.30 and 50.04 per cent at $3,7,11$ and 15 days after second spray, respectively. Thiamethoxam 25\% WG, Acetamiprid 20\% SP and Spinosad $45 \%$ SC were also found effective against aphid population. In insecticides the lowest efficacy against cabbage aphid was recorded in the plot treated with Novaluron $10 \%$ EC. Just like after first spray among the botanicals Neem leaf extract + Cow urine again observed highly effective against aphid i.e. 131.67, 137.33, 141.33 and 144.67 aphids per plant and the per cent change over control was $10.19,10.3,8.91$ and 8.59 per cent at 3, 7 , 11 and 15 days after second spray, respectively. Among all the treatments after control Lantana leaf extract + Cow urine was observed less effective (i.e. 175.00, 181.67, 185.00 and 188.67 aphids per plant at 3, 7, 11 and 15 days after spray, respectively) against cabbage aphid. 
Table No. 4: Efficacy of different insecticides and botanicals against cabbage aphid after second spray.

\begin{tabular}{|c|c|c|c|c|c|c|}
\hline \multirow{3}{*}{ T. No. } & \multirow{3}{*}{ Treatments } & \multirow{3}{*}{$\begin{array}{l}\text { Dose } \\
(\%)\end{array}$} & \multirow{2}{*}{\multicolumn{4}{|c|}{$\begin{array}{l}\text { No. of cabbage aphid/ plant } \\
\text { After } 2^{\text {nd }} \text { application }\end{array}$}} \\
\hline & & & & & & \\
\hline & & & 3 DAS & 7 DAS & 11 DAS & 15 DAS \\
\hline $\mathrm{T}_{1}$ & Control & - & $\begin{array}{l}268.00 \\
(16.40)\end{array}$ & $\begin{array}{l}280.00 \\
(16.76)\end{array}$ & $\begin{array}{l}283.67 \\
(16.87)\end{array}$ & $\begin{array}{l}287.33 \\
(16.98)\end{array}$ \\
\hline $\mathrm{T}_{2}$ & Neem leaf extract + Cow urine & 5 & $\begin{array}{l}131.67^{*} \\
(11.51)\end{array}$ & $\begin{array}{l}137.33^{*} \\
(11.76)\end{array}$ & $\begin{array}{l}141.33^{*} \\
(11.93)\end{array}$ & $\begin{array}{l}144.67 * \\
(12.03)\end{array}$ \\
\hline $\mathrm{T}_{3}$ & Lantana leaf extract + Cow urine & 5 & $\begin{array}{l}175.00^{*} \\
(13.27)\end{array}$ & $\begin{array}{l}181.67^{*} \\
(13.51)\end{array}$ & $\begin{array}{l}185.00^{*} \\
(13.63)\end{array}$ & $\begin{array}{l}188.67 * \\
(13.77)\end{array}$ \\
\hline $\mathrm{T}_{4}$ & Parthaniun leaf extract + Cow urine & 5 & $\begin{array}{l}170.33^{*} \\
(13.08)\end{array}$ & $\begin{array}{l}176.33^{*} \\
(13.31)\end{array}$ & $\begin{array}{l}180.33^{*} \\
(13.46)\end{array}$ & $\begin{array}{l}184.91 * \\
(13.63)\end{array}$ \\
\hline $\mathrm{T}_{5}$ & Eupatorium leaf extract + Cow urine & 5 & $\begin{array}{l}157.67 * \\
(12.55)\end{array}$ & $\begin{array}{l}163.78^{*} \\
(12.79)\end{array}$ & $\begin{array}{l}165.78^{*} \\
(12.91)\end{array}$ & $\begin{array}{l}168.00 * \\
(13.00)\end{array}$ \\
\hline $\mathrm{T}_{6}$ & Novaluron $10 \%$ EC & 0.0075 & $\begin{array}{l}92.64^{*} \\
(9.67)\end{array}$ & $\begin{array}{l}86.33^{*} \\
(9.34)\end{array}$ & $\begin{array}{l}91.67 * \\
(9.62)\end{array}$ & $\begin{array}{l}92.26^{*} \\
(9.65)\end{array}$ \\
\hline $\mathrm{T}_{7}$ & Thiamethoxam $25 \% \mathrm{WG}$ & 0.0025 & $\begin{array}{l}62.40 * \\
(7.96)\end{array}$ & $\begin{array}{l}48.40^{*} \\
(7.02)\end{array}$ & $\begin{array}{l}55.33^{*} \\
(7.51)\end{array}$ & $\begin{array}{l}64.33^{*} \\
(8.08)\end{array}$ \\
\hline $\mathrm{T}_{8}$ & Imidacloprid $17.8 \% \mathrm{SL}$ & 0.0356 & $\begin{array}{l}52.33^{*} \\
(7.30)\end{array}$ & $\begin{array}{l}32.92 * \\
(5.82)\end{array}$ & $\begin{array}{l}38.89^{*} \\
(6.32)\end{array}$ & $\begin{array}{l}49.99^{*} \\
(7.14)\end{array}$ \\
\hline $\mathrm{T}_{9}$ & Spinosad 45\% SC & 0.0144 & $\begin{array}{l}86.00 * \\
(9.32)\end{array}$ & $\begin{array}{l}78.15^{*} \\
(8.89)\end{array}$ & $\begin{array}{l}84.60^{*} \\
(9.25)\end{array}$ & $\begin{array}{l}91.00^{*} \\
(9.59)\end{array}$ \\
\hline $\mathrm{T}$ & Acetamiprid $20 \%$ SP & 0.002 & $\begin{array}{l}82.67 * \\
(9.14)\end{array}$ & $\begin{array}{l}71.67 * \\
(8.52)\end{array}$ & $\begin{array}{l}78.67^{*} \\
(8.92)\end{array}$ & $\begin{array}{l}88.67^{*} \\
(9.47)\end{array}$ \\
\hline & $\mathrm{SE}(\mathrm{d})$ & - & 1.20 & 1.14 & 0.87 & 1.01 \\
\hline & C.D.(0.05) & - & 2.54 & 2.41 & 1.84 & 2.13 \\
\hline
\end{tabular}

DAS- days after spray, ()$=$ Values in parentheses are square root transformed value, *Significant at $5 \%$ level of significance compared with control. 
Table No. 5: Change in cabbage aphid population after first spray after second spray.

\begin{tabular}{|c|c|c|c|c|c|c|}
\hline \multirow[t]{2}{*}{ T.No. } & \multirow[t]{2}{*}{ Treatments } & \multirow{2}{*}{$\begin{array}{c}\text { Dose } \\
(\%)\end{array}$} & \multicolumn{4}{|c|}{$\begin{array}{l}\text { Per cent change in aphid population } \\
\text { after second spray }\end{array}$} \\
\hline & & & 3 DAS & 7 DAS & 11 DAS & 15 DAS \\
\hline $\mathrm{T}_{1}$ & Control & - & $\begin{array}{c}0.00 \\
(0.00)\end{array}$ & $\begin{array}{c}0.00 \\
(0.00)\end{array}$ & $\begin{array}{c}0.00 \\
(0.00)\end{array}$ & $\begin{array}{c}0.00 \\
(0.00)\end{array}$ \\
\hline $\mathrm{T}_{2}$ & Neem leaf extract + Cow urine & 5 & $\begin{array}{l}10.19^{*} \\
(18.60)\end{array}$ & $\begin{array}{l}10.33^{*} \\
(18.74)\end{array}$ & $\begin{array}{c}8.91^{*} \\
(17.36)\end{array}$ & $\begin{array}{l}8.59 * \\
(17.02)\end{array}$ \\
\hline $\mathrm{T}_{3}$ & Lantana leaf extract + Cow urine & 5 & $\begin{array}{l}6.63^{*} \\
(14.90)\end{array}$ & $\begin{array}{l}7.56^{*} \\
(15.90)\end{array}$ & $\begin{array}{c}7.08^{*} \\
(15.38)\end{array}$ & $\begin{array}{l}6.02 * \\
(14.19)\end{array}$ \\
\hline $\mathrm{T}_{4}$ & Parthaniun leaf extract + Cow urine & 5 & $\begin{array}{l}08.59^{*} \\
(17.03)\end{array}$ & $\begin{array}{l}9.30 * \\
(17.73)\end{array}$ & $\begin{array}{c}8.57^{*} \\
(16.96)\end{array}$ & $\begin{array}{l}7.63 * \\
(16.03) \\
\end{array}$ \\
\hline $\mathrm{T}_{5}$ & Eupatorium leaf extract + Cow urine & 5 & $\begin{array}{l}08.17^{*} \\
(16.58)\end{array}$ & $\begin{array}{l}8.87 * \\
(17.31)\end{array}$ & $\begin{array}{c}8.39 * \\
(16.80)\end{array}$ & $\begin{array}{c}7.51^{*} \\
(15.88)\end{array}$ \\
\hline $\mathrm{T}_{6}$ & Novaluron $10 \%$ EC & 0.0075 & $\begin{array}{l}13.30^{*} \\
(21.36)\end{array}$ & $\begin{array}{l}22.67 * \\
(28.41)\end{array}$ & $\begin{array}{l}19.14^{*} \\
(25.93)\end{array}$ & $\begin{array}{l}19.12^{*} \\
(25.92)\end{array}$ \\
\hline $\mathrm{T}_{7}$ & Thiamethoxam 25\% WG & 0.0025 & $\begin{array}{l}34.47 * \\
(35.93)\end{array}$ & $\begin{array}{l}51.34^{*} \\
(45.75)\end{array}$ & $\begin{array}{l}44.47^{*} \\
(41.80)\end{array}$ & $\begin{array}{l}37.02^{*} \\
(37.45)\end{array}$ \\
\hline $\mathrm{T}_{8}$ & Imidacloprid $17.8 \% \mathrm{SL}$ & 0.0356 & $\begin{array}{l}43.93^{*} \\
(41.49)\end{array}$ & $\begin{array}{l}66.23^{*} \\
(54.45)\end{array}$ & $\begin{array}{l}60.30^{*} \\
(50.93)\end{array}$ & $\begin{array}{l}50.04^{*} \\
(45.01)\end{array}$ \\
\hline $\mathrm{T}_{9}$ & Spinosad $45 \%$ SC & 0.0144 & $\begin{array}{l}18.67^{*} \\
(25.58)\end{array}$ & $\begin{array}{l}29.26^{*} \\
(32.73)\end{array}$ & $\begin{array}{l}24.39^{*} \\
(29.58)\end{array}$ & $\begin{array}{l}19.36^{*} \\
(26.09)\end{array}$ \\
\hline $\mathrm{T}_{10}$ & Acetamiprid $20 \%$ SP & 0.002 & $\begin{array}{l}19.27 * \\
(26.02)\end{array}$ & $\begin{array}{l}33.02^{*} \\
(35.05)\end{array}$ & $\begin{array}{l}27.45^{*} \\
(31.58)\end{array}$ & $\begin{array}{l}19.37^{*} \\
(26.10)\end{array}$ \\
\hline & $\mathrm{SE}(\mathrm{d})$ & - & 0.65 & 1.10 & 0.88 & 0.69 \\
\hline & C.D.(0.05) & - & 1.39 & 2.32 & 1.85 & 1.46 \\
\hline
\end{tabular}

DAS- days after spray, ( ) = Values in parentheses are angular transformed value

After both sprays the efficacy was recorded to be minimum in plots treated with Imidacloprid $17.8 \% \mathrm{SL}$ followed by Thiamethoxam 25\% WG, Acetamiprid 20\% SP and Spinosad $45 \%$ SC. The present findings are also correlated with observation made by Ghosal et al. (2013) who reported that Imidacloprid to be the best effective insecticidal treatment against the aphids with 84.54 per cent protection over control followed by Thiamethoxam and Acetamiprid. The present investigation on neonicotinoid molecules against aphid also appeared similar to the finding of Mishra (2002) and Jafarpour et al., (2011) who reported that neonicotinoids have good ingestion toxicity in comparison with others. Jadhav et al. (2016) also observed that

Imidacloprid was best insecticide as compared to Thiamethoxam and sAcetamiprid. Varghese and Mathew (2012) concluded that Imidacloprid, Thiamethoxam and Acetamiprid were highly effective against aphid when compared with Spinosad.

Among the botanicals, after both sprays, Neem leaf extract + Cow urine caused maximum per cent reduction in aphid population followed by Parthaniun leaf extract + Cow urine, Eupatorium leaf 
extract + Cow urine and Lantana leaf extract + Cow urine. These results are similar to the findings of Sable and Kushwaha (2014) who reported that Neem leaf extract and Parthaniun leaf extract were more effective than Lantana leaf extract. Ali and Zedan (2015) also reported that Eupatorium leaf extract causes maximum mortality on $B$. brassicae than Lantana leaf extract.

\section{REFERENCES}

Ali, R. A. E. and Zedan, O. A. A. 2015. Selectivity of certain insecticides for controlling the cabbage aphid Brevicoryne brassicae and their effect on some predatory insects on cauliflower fields in el-minia region-upper egypt. Journal of Plant Protection and Path.6 (10): 1427- 1437.

Dubey, A., Gupta, R. and Chandel, B. S. 2004. Efficacy of Acorus calamus, Vitex negundo and Ageratum conyzoides against Tobacco caterpillar, Spilarctia obliqua (Walker). Indian Journal of Entomology. 66: 238-240.

Garratt, J. and Kennedy, A. 2006. Use of models to assess the reduction in contamination of water bodies by agricultural pesticides through the implementation of policy instruments: a case study of the Voluntary Initiative in the UK. Pest Management Science. 62: 1138-1149.

Ghosal, A., Chatterjee, M. L. and Bhattacharya, A. 2013. Bio-efficacy of neonicotinoids against Aphis gossypii
Glover of okra. Journal of Crop and Weed. 9(2):181-184.

Gupta, M. P. 2005. Efficacy of neem in combination with cow urine against mustard aphid and its effect on coccinellid predators. Inidian of Natural Products and Resources. 4(2): 102-106.

Hasan, M. R. and Solaiman, A. H. M. 2012. Efficacy of organic and organic fertilizer on the growth of Brassica oleracea L. (cabbage). International Journal of Agriculture and Crop Sciences. 4(3): 128138

Iwasa, T., Motoyama, N., Ambrose, J. T. and Roe, R. M. 2004. Mechanism for the differential toxicity of neonicotinoid insecticides in the honey bee, Apis mellifera. Crop Protection. 23: 371-378.

Jadhav, Y. T., Mane, S. R. and Shinde, D. S. 2016. Effect of different newer pesticides on aphid population of summer okra. International Journal. 2(9): 418-423.

Jafarpour A A, Garjan S A, Mani S, Mahjoub S M \& Mohammad M. 2011. Toxic effects of neonicotinoid on nymph \& adult of the sunn pest Eurygaster intergriceps Puton (hemiptera: Scutelleridae). Entomologica Sinica. 54(8): 938-942.s

Khan, I. A., Ahmad, M., Akbar, R., Hussain, S., Muhammad, S. and Fayaz, W. 2015. A study on Losses due to Brevicoryne brassicae in different Brassica genotypes under screen house conditions. Journal of Entomology. 3(6): 16-19. 
Lal, O. P. 1975. A compendium of insect pest of vegetables in India. Bulletin of Entomology. 16: 31-56.

Maienfisch, P., Gsell, L. and Rindhisbacher, A. 1999. Synthesis and insecticidal activity of CGA 293343-a novel board-spectrum insecticide. Pesticide Science. 55:351-354.

Mishra, H. P. 2002. Field evaluation of some newer insecticides against aphids (Aphid gossypii) and jassids (Amrasca biguttula biguttula) on okra. Indian Journal of Entomology. 64: 80-84.

Opfer, P. and McGrath, D. 2013. Oregon vegetables,cabbage aphid and green peach aphid. Department Horticulture Oregon State University, Carvallis. International Organisation for Biological and Integrated Control / WPRS Bulletin. 17(8): 73-182.

Prasad, S. K. 1963. Quantitative estimation of damage to crucifer caused by cabbageworm, cabbagelooper, diamondback moth and cabbage aphid. Indian Journal of Entomology. 25:242-259.
Sable, M. and Kushwaha, R. K. 2014. Efficacy of different plant leaf extract against mustard aphid lipaphis erysimi (kalt). Journal of Industrial Pollution Control. 30(2): 231-233.

Sharma, A., Gupta, R. and Kanwar, R. 2009. Larvicidal effect of some plant extracts against Spodoptera litura (Fab.) and Pieris brassicae (Linn.) Journal of Entomological Research. 33: 213-218.

Sood, A. K., Mehta, P. K. and Parmar, S. 2000. Evaluation of plant extracts against cabbage aphid, Brevicoryne brassicae (Linn.). Insect Environment. 6(2): 87-89.

Vanitha S M, Chaurasia S N S, Singh P M and Naik P S. 2013. Vegetable Statistics Technical. Indian Institute of Vegetable Research. 51: 1-250.

Varghese, T. S. and Mathew, T. B. 2012. Evaluation of newer insecticides against chilli aphids and their effect on natural enemies. Pest Management in Horticultural Ecosystems. 18(1): 114-117. 